\title{
Los programas universitarios para mayores como agente de educación para el desarrollo
}

\author{
José-Luis Rodríguez-Díez¹, Victoria Pérez-de-Guzmán² \\ Fecha: Recibido noviembre 15 de 2014 - Aceptado diciembre 12 de 2014
}

\begin{abstract}
Resumen
Desarrollo, educación y participación han formado parte de la historia de la humanidad. Con esta investigación se pretende concienciar cómo los programas universitarios para mayores y, por ende, la formación a lo largo de la vida, deben actuar como agentes de educación para el desarrollo. Para ello la metodología seguida está centrada principalmente en el ámbito descriptivo, basado en un enfoque diacrónico a través de un muestreo teórico de fuentes documentales. El propósito del mismo es articular la dimensión social y pedagógica de las temáticas que se ha desarrollado en el presente estudio. Como resultados del recorrido se puede afirmar que, tanto la educación para el desarrollo como los programas universitarios para mayores tienen objetivos comunes y que el fin último de ambos es mejorar la calidad de vida de las personas y de la sociedad en general.
\end{abstract}

Palabras clave: calidad de vida; aprendizaje a lo largo de la vida; educación para el desarrollo; adultos mayores; educación no formal; educación permanente.

\section{University programs for seniors as agents for the Education for Development}

\begin{abstract}
Development, education and participation have been part of the history of humanity. With this research we want to raise awareness how University Programs for seniors and therefore training throughout life, act as agents for the Education for Development For this, the methodology followed is focused mainly on the descriptive level, based on a diachronic approach through theoretical sampling of documentary sources. The purpose is to articulate the social and educational aspect of the themes that have developed in the present study. As a result of our journey, we found that both the Education for Development and the University Programs for Senior have common objectives, and the main reason is to improve the quality of life of people and society in general.
\end{abstract}

Keywords: quality of life, longlife learning, education for development; seniors; non-formal education; continuing education.

1Doctorando a través del Departamento de Educación y Psicología social. Docente Investigador de la Universidad Pablo Olavide de Sevilla (España).jlrdiez@gmail.com

2Doctora en Pedagogía social. Docente Investigadora de la Universidad Pablo de Olavide de Sevilla (España). mvperpuy@upo.es 


\section{Introducción}

La población mundial envejece, no solo en el primer mundo, sino en todo el planeta de forma imparable y en un proceso sin precedentes en la historia de la humanidad (Ceberio, 2010).

Una de cada cinco personas en los países desarrollados tiene más de 60 años y las predicciones de la Organización de las Naciones Unidas (ONU) indican que en 2050 serán una de cada tres. España está entre los países con mayor esperanza de vida al nacer.

El aumento de la esperanza de vida, no solo es un dato demográfico, la mayoría de los mayores, actualmente, presenta un nivel de bienestar equiparable e incluso mejor que el de muchos jóvenes, y por supuesto inimaginable hace tan solo cuatro décadas (Pérez, 2003).

Este nivel de bienestar propicia, según la Encuesta mayores 2010 del Instituto de Mayores y Servicios Sociales de España (IMSERSO), que un $60 \%$ de las personas inicie nuevas actividades tras la jubilación. Una de las demandas de esta nueva generación de adultos mayores es una formación universitaria de calidad, más allá de la formación informal.

La formación a lo largo de la vida es una necesidad que viene determinada por diversos factores (Limón, 2007) y se debe estar preparados para dar la respuesta adecuada a un mundo donde la tecnología, el estilo de vida, el mundo laboral, las relaciones humanas, las costumbres familiares, la interculturalidad, las exigencias de la mundialización, los valores, etc., están cambiando constantemente y es difícil anticipar cómo se va a configurar la realidad nueva que se abre paso. Estos hechos van creando en el ser humano, y sobre todo en los adultos, incertidumbre, intranquilidad y desasosiego (Rodríguez-Díez, Amador, Pérez-De-Guzmán \& Esteban, 2012).

En la historia de la humanidad ninguna generación ha tenido que adaptarse a tantos cambios en tan poco tiempo como los actuales adultos mayores. Los grandes cambios sociales y familiares, al espectacular avance de la sociedad de la información y la comunicación y, en nuestro país, la incursión de la mujer y su participación activa en la vida social, política y económica entre otros, son hitos que necesitan de una adaptación por parte de las personas mayores.

Sucapacidad deadaptación incide claramenteen su calidad de vida y en el logro de un envejecimiento activo. Sin embargo ¿qué entendemos por envejecimiento activo? Según el Libro Blanco del Envejecimiento Activo (VV. AA, 2010).

\begin{abstract}
El envejecimiento debería ser contemplado como un proceso que a todos nos afecta, que requiere modulaciones sociales y respuestas adaptadas. [...] Por ello se hace preciso adoptar hábitos de vida saludable, animarse a participar, a vivir, a opinar. Adoptar un estilo de vida presidido por la libertad de elegir, de disponer del patrimonio para el futuro, de decidir en nuestro entorno, de ejercer una necesaria solidaridad ciudadana, de crecer cada día como personas individuales y miembros de la sociedad (p.17).
\end{abstract}

Esta definición hace alusión claramente a los conceptos barajados en Educación para el Desarrollo.

Como afirma Argibay, Celorio y Celorio (1997, p.5): "El desarrollo, la educación y la participación siempre han formado parte de la historia de la humanidad". Por tanto, a tenor de esta definición, los conceptos calidad de vida y educación para el desarrollo también deben estar íntimamente unidos, pues no es entendible el uno sin el otro.

La actual concepción de la educación para el desarrollo (EpD) (la quinta generación) se dirige a la educación para la ciudadanía global o mundial (Boni, 2005). El informe Delors (1996) establecía que los pilares fundamentales de la Educación para el siglo XXI se sustentan sobre cuatro enseñanzas básicas (Delors, 1996), estas eran:

1. Aprender a hacer

2. Aprender a aprender 
BÚSQUEDA - Julio / Diciembre de 2014 - No. 13 (32 - 40)

3. Aprender a ser

4. Aprender a vivir juntos

Más allá de esto, la $5^{a}$ generación de la Educación para el Desarrollo considera la educación como un bien común de la humanidad estableciendo la necesidad de las personas como el objetivo del desarrollo y no la necesidad del mercado.

Por tanto, entendiendo que la formación a lo largo de la vida (Long Life Learning) se ha convertido en una necesidad de los seres humanos, consideramos que ésta y en concreto los Programas Universitarios para Mayores (PUM) pueden actuar como agentes de Educación para el Desarrollo procurando la calidad de vida de las personas adultas mayores, en este caso.

\section{Metodología}

La metodología seguida está centrada principalmente en un método descriptivo basado en un enfoque diacrónico y mediante un muestreo teórico de fuentes documentales. El propósito metodológico es articular la dimensión social, política y pedagógica de las temáticas estudiadas.

Los estudios descriptivos buscan especificar las propiedades importantes de personas, grupos, comunidades o cualquier otro fenómeno que sea sometido a análisis (Dankhe, 1986, citado por Hernández, Fernández \& Bautista, 1998) y así mismo, miden o evalúan diversos aspectos, dimensiones o componentes del fenómeno o fenómenos a investigar. Desde el punto de vista científico, describir es medir. Esto es, en un estudio descriptivo se selecciona una serie de cuestiones y se mide cada una de ellas independientemente, para así -y valga la redundancia- describir lo que se investiga.

Los programas universitarios para mayores como agentes de educación para el desarrollo

Aunque resulta muy difícil extrapolar los objetivos, metodología y resultados de los distintos programas universitarios para mayores implementados en las distintas universidades ya que cada programa está diseñado dependiendo del propio ideario del Centro y del alumnado al que va dirigido, si podemos establecer una serie de objetivos generales (Amador, 2009), entre los que se encuentran:

- Contribuir al desarrollo personal desde una propuesta científica y cultural.

- Procurar un espacio natural para las relaciones sociales.

- Promover la intergeneracionalidad, con la finalidad de desbancar los estereotipos sobre la vejez y la juventud.

- Abrir un nuevo campo a los investigadores universitarios.

Podríamos establecer un paralelismo entre estos objetivos y las dimensiones de la Educación para el Desarrollo. Las cuatro dimensiones de ésta son: Sensibilización, Educación-Formación, Investigación, e Incidencia política y movilización social.

Estascuatro dimensionesestáninterrelacionadas entre sí, así como los objetivos de los Programas Universitarios para Mayores también lo están para dar lugar a un sin fin de actuaciones.

Así podríamos establecer la siguiente interrelación (figura 1):

- Sensibilización, esta dimensión estaría relacionada con el objetivo de promover la intergeneracionalidad ya que la finalidad es desbancar los estereotipos sobre la vejez y la juventud y entendemos que esto es sensibilizar.

- Educación-Formación, los PUM tratan de contribuir al desarrollo personal desde una propuesta científica y cultural.

- Investigación, uno de los objetivos comunes de los PUM es abrir un nuevo campo a los investigadores universitarios. 
Figura 1. Relacion entre los objetivos de los Programas Universitarios para Mayores y las dimensiones de la Educación para el Desarrollo

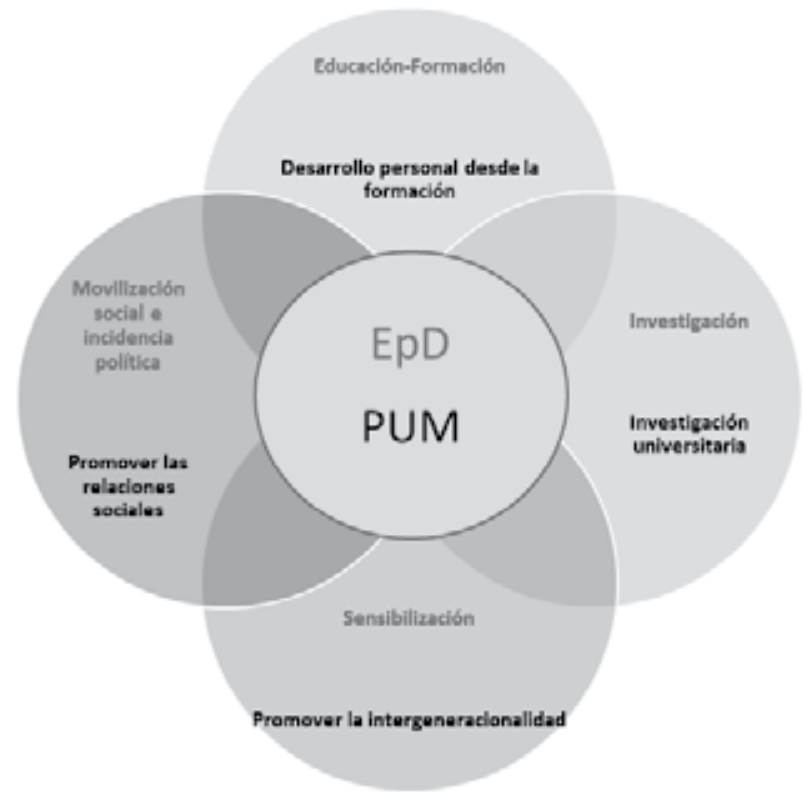

Fuente: Elaboración propia

- Incidencia política y movilización social, así mismo los PUM procuran un espacio natural para las relaciones sociales, las relaciones sociales promueven por sí mismas la movilización y por tanto, la incidencia política.

Consideramos que tanto la Educación para el Desarrollo como los Programas Universitarios para Mayores tienen como finalidad mejorar la calidad de vida de las personas

La dimensión pedagógica de la Educación para el Desarrollo

La dimensión pedagógica de la Educación para el Desarrollo está dentro de su propio enunciado. En palabras de Argibay y Celorio (2005), "Presupone un acto volitivo, estructurado, basado en técnicas de construcción del saber y métodos de formación que conseguirán transformar a las personas en promotores del cambio social" (p.75).

El mundo actual es una realidad global. Por tanto, los problemas, al igual que las soluciones, deben ser globales. Los avances en las nuevas tecnologías han hecho al mundo cada vez más pequeño; sabemos lo que pasa en la otra punta del globo en tiempo real, sin embargo cada vez estamos más solos. Lo que pasa al otro lado de la pantalla no parece real, nos hemos convertido en autómatas devoradores de imágenes. El problema es que esas imágenes no empapan nuestra conciencia. La sociedad es cada vez más individualista.

En esta realidad se hace necesaria la Educación para el Desarrollo, hay que despertar a la mujer o al hombre que llevamos dentro. En definitiva se hace necesario que el ser humano vuelva a ser humano. Hay que generar conciencias críticas, responsabilidad, activismo, compromiso y participación en el nuevo ser humano.

Esta conciencia se forma a través de la formación. La formación a lo largo de la vida y por ende los Programas Universitarios para Mayores generan conciencias críticas, responsabilidad, activismo, compromiso y participación en su alumnado (Blázquez, 2002; Castellón, Gómez \& Martos, 2004). 
BÚSQUEDA - Julio / Diciembre de 2014 - No. 13 (32 - 40)

\section{Los agentes de Educación para el Desarrollo}

Las instituciones públicas, los medios de comunicación, las empresas, las cooperativas, los bancos o cajas de ahorro, los sindicatos, las universidades, los centros educativos y los movimientos sociales, entre otros, pueden ser agentes promotores de acciones de Educación para el Desarrollo (Argibay \& Celorio, 2005).

Por tanto, existen múltiples instituciones públicas y privadas que directa o indirectamente actúan como agentes de Educación para el Desarrollo.

Independientemente del agente y del contexto donde se desarrolle la acción (educación informal, no formal o formal), para que consideremos una acción formativa como de Educación para el Desarrollo debe mantener las siguientes características entre las que se encuentran (Celorio, 1995):

- Es una educación global.

- Es una educación emancipadora.

- Es una educación comprometida.

- Tiene una epistemología crítica.

- Una visión holística de los problemas.

- Constructiva en el aprendizaje individual y social.

- Es crítica con el currículum.

- Es una educación en valores.

- Los valores como sistemas.

- Comprensión crítica de los valores.

- La contradicción y el conflicto en los valores.

- Distintas visiones valorativas.

Las universidades constituyen un espacio privilegiado de investigación, reflexión y formación en y para la Educación para el Desarrollo, por tanto deben promoverse acciones coordinadas o conjuntas entre ellas y con el resto de actores (Coordinadora de ONGD España, 2005).

Las propuestas para el nuevo Espacio Europeo de Educación Superior (EEES), apoyan la importancia de la formación global e integral, y resaltan el compromiso ético y el reconocimiento de la interculturalidad, buscando el equilibrio entre la formación personal y ciudadana.
Por tanto, los Programas universitarios para Mayores, como parte integrante de la educación formal promovida desde la universidad, podemos considerarlos como agentes de Educación para el Desarrollo.

\section{La formación a lo largo de la vida}

La formación a lo largo de la vida es una necesidad que viene determinada por diversos factores tales como: exigencias democráticas, grandes cambios sociales, crisis de las ideologías y de los modelos de vida, democratización de la educación y de la cultura, las nuevas tecnologías de la información y la comunicación, incremento acelerado del conocimiento, aumento del tiempo libre y del ocio y envejecimiento de la población (Limón, 2007).

El concepto de formación a lo largo de la vida ha pasado por diferentes fases hasta llegar a la situación actual. Cuatro son los modelos en los que se puede resumir esta trayectoria (Moody, 1976):

- En primer lugar, nos encontramos el modelo de rechazo. En las épocas en las que la sociedad rechazaba la vejez, desde el punto de vista educativo, si los mayores no producían beneficios no tenía sentido invertir en la educación de este sector de la población.

- En segundo lugar, surge el modelo de los servicios sociales, que presenta un carácter asistencial, por tanto el terreno educativo se limita a tener entretenidos a los mayores.

- En tercer lugar, el modelo de participación propone la intervención de los mayores en actividades socioeducativas.

- Y en cuarto lugar, el modelo de autorrealización, donde la educación actúa como vehículo para el crecimiento del individuo, independientemente de su edad.

Según el Libro Blanco del Envejecimiento Activo (W. AA., 2010) se entiende por formación permanente la capacidad de aprendizaje que tienen las personas de todas las edades del ciclo vital. 
Hasta llegar a la definición anteriormente expuesta, podríamos señalar diferentes etapas en el desarrollo del concepto (Pérez Serrano, 2001):

1) Las Conferencias Mundiales de Educación de Adultos, organizadas por la UNESCO, que ponen su acento en la perspectiva remedial.

2) La aparición del concepto Educación Permanente que establece que no existe una barrera infranqueable entre educación de jóvenes y educación de adultos y personas mayores y se desarrolla en tres principios básicos (Calderón, 2010; Escarbajal de Haro \& Martínez de Miguel, 2012): el principio de participación, el principio de globalidad y el principio de igualdad de oportunidades.

3) Se concibe la educación como extensiva a lo largo de la vida humana.
Los programas universitarios para mayores se están convirtiendo en uno de los pilares de la formación a lo largo de la vida.

\section{Los programas universitarios para personas mayores}

Las universidades de mayores, aunque inicialmente se denominaron de la tercera edad, surgen en Europa en 1973 propiciadas por el profesor Pierre Vellasen en la Universidad de Toulouse (Francia) (Requejo, 1999; VV. AA. 2010).

Este tipo de programas universitarios ha pasado por tres etapas desde su nacimiento (Lemiex, 1998) (ver tabla 1).

Tabla 1. Evolución de los programas para mayores

\begin{tabular}{|l|l|l|}
\hline Etapas & \multicolumn{1}{|c|}{ Actividad educativa } & \multicolumn{1}{|c|}{ Finalidad/Objetivos } \\
\hline $1^{\text {a Etapa }}$ & $\begin{array}{l}\text { Servicios educativos concebidos como } \\
\text { programas culturales de tiempo libre. }\end{array}$ & $\begin{array}{l}\text { Entretener. } \\
\text { Favorecer las relaciones sociales entre } \\
\text { las personas mayores. }\end{array}$ \\
\hline $2^{\text {a Etapa }}$ & $\begin{array}{l}\text { Actividades educativas para la participación } \\
\text { mayojora de los conocimientos de los }\end{array}$ & $\begin{array}{l}\text { Intervención de los mayores en los } \\
\text { problemas sociales existentes. }\end{array}$ \\
\hline $3^{\text {a Etapa }}$ & $\begin{array}{l}\text { Programas educativos reglados, con Plan de Estudios propio, con todas las } \\
\text { características propias de la enseñanza superior y, generalmente, propuestos desde } \\
\text { las Ciencias de la Educación. }\end{array}$ \\
\hline
\end{tabular}

Fuente: VV. AA. (2010)

Dos tradiciones fundamentales, la francesa y la británica son las que inspiran el desarrollo de las distintas experiencias en este sentido, que al mismo tiempo dan lugar a dos modelos de "universidad" diferentes:

- Según la tradición francesa: coincide con lo que se denominaría un modelo formal de universidad. Se trata del primer modelo desarrollado y se corresponde propiamente con los llamados "programas universitarios para mayores". Son programas creados y tutelados por las propias universidades e impartidos por los profesores de dicha institución. Se estructuran generalmente en cursos académicos. Este modelo parece ser el más extendido en la mayor parte de Europa.

- Según la tradición británica: se desarrolla un modelo que no busca "ni la afiliación ni el patrocinio de la Universidad"; de hecho, los programas que surgen no necesariamente se originan en el contexto universitario y las experiencias de aprendizaje tiene lugar en distintos espacios. Se establece bajo los principios de autoayuda y autosuficiencia y priman los ideales de 
BÚSQUEDA - Julio / Diciembre de 2014 - No. 13 (32 - 40)

participación y democracia intelectual. En este caso la denominación "universidad" se refiere a una comunidad de sabios/eruditos que buscan el conocimiento y la verdad en sí mismos. Además son los propios miembros de esta universidad, las personas mayores, las que desarrollan tanto el rol de alumno como el de profesor. Este modelo parece tener una mayor difusión en Norteamérica que en Europa.

En España esta experiencia se inaugura con la creación en 1978 de las Aulas de la Tercera Edad que, aunque no se consiente su denominación como universidades con todo lo que este término engloba, sí se consigue su constitución como una serie de centros en red dependientes del Ministerio de Cultura de aquel entonces. En algunos lugares, como en Cataluña, las antiguas Aulas para Mayores (cuya organización tenía su origen en asociaciones, comunidades o ayuntamientos), se consigue unos años más tarde (durante los '80) una tutela universitaria de sus programas educativos. No obstante, habrá que esperar hasta los años 90 del siglo pasado para que la universidad abriera las puertas a las personas mayores.

Concretamente, las universidades pioneras fueron las de Alcalá de Henares (1992), Salamanca (1993) y Granada (1994), entre otras. El enfoque fundamental en el territorio español en el desarrollo de programas universitarios para mayores viene caracterizado por el diseño de programas formales que se implementan bajo distintas denominaciones: universidad de la tercera edad, aula permanente, universidad de la experiencia, universidad para mayores, etc.

Pasamos a realizar una exposición cronológica del orden de implementación de estos programas en las universidades españolas.

Los primeros programas se desarrollaron en las Universidades de Lérida y Gerona (1982), Alcalá de Henares (1990) y Salamanca (1993), "aunque no fue hasta el año 1999, nombrado Año Internacional de las Personas Mayores, cuando comenzaron a proliferar los programas universitarios para mayores" (Villaplana, 2010, p.4)
Aunque resulta muy difícil extrapolar los objetivos, metodología y resultados de los distintos Programas Universitarios para Mayores implementados en las distintas Universidades ya que cada programa está diseñado dependiendo del propio ideario del Centro y del alumnado al que va dirigido, si podemos establecer una serie de objetivos generales (Amador, 2009), entre los que se encuentran:

- Contribuir al desarrollo personal desde una propuesta científica y cultural.

- Procurar un espacio natural para las relaciones sociales.

- Promover la intergeneracionalidad, con la finalidad de desbancar los estereotipos sobre la vejez y la juventud.

- Abrir un nuevo campo a los investigadores universitarios.

En España, los Programas Universitarios para Mayores han desarrollado un crecimiento exponencial desde su creación. Según los últimos datos que se disponen de la Asociación Estatal de Programas Universitarios para Mayores (AEPUM), en el curso 2012-13, un total de 40.072 personas accedieron a estos programas (14.113 hombres y 25.959 mujeres). Hay que señalar que otras 30 universidades españolas que no están asociadas a AEPUM imparten estos programas, por lo que el número total de alumnos en este tipo de enseñanzas debe ser bastante superior al facilitado anteriormente.

En definitiva, las Aulas de Mayores son un elemento importante en educación que contribuyen a elevar la calidad de vida de nuestros mayores (Castellón, Gómez \& Martos, 2004) y así mismo pueden actuar como agentes de Educación para el Desarrollo.

\section{Conclusiones}

Numerosas investigaciones han demostrado el impacto positivo que tienen los programas 
universitarios paramayores sobre la calidad de vida de su alumnado y en el aumento y fortalecimiento de su red social. En el estudio de Vives, Macías \& Orte (2004) se concluye que los PUM tienen tres consecuencias sobre sus participantes:

- El aumento de su autoestima y una visión más positiva de su autoconcepto.

- El aumento y fortalecimiento de su red social y

- Un enriquecimiento personal y cultural.

Por otro lado, Fernández, Macías y Orte (2009) concluyen que el alumnado del programa para mayores de la Universidad de las Islas Baleares presenta mejores indicadores de salud y de calidad de vida percibida en comparación con los datos de la población general de adultos mayores.

Destacar también que los PUM representan una posibilidad para superar la brecha digital (Fernández, Macías, Mas \& Orte, 2008, pp. 233244) y su alumnado hace uso de las nuevas tecnologías con mayor frecuencia que la población general de su grupo etario.

Los Programas Universitarios para Mayores son una potente herramienta con respecto a la Educación para el Desarrollo, y desde la Universidad se debe animar a que los mayores participen en actividades de voluntariado dirigido a la EpD ya que como afirma Carbonell y Medina (2006), la incorporación como voluntarios de las personas mayores supone importantes ventajas para toda la sociedad y para los propios mayores. Estas ventajas las podemos clasificar en:

- Ventajas sociales, al concienciar a la población de la importancia de las personas mayores, para mejorar su imagen social.

- Ventajas para las organizaciones, al aumentar el número de voluntarios con una gran experiencia y dedicación.

- Ventajas para los voluntarios, al favorecer las relaciones intergeneracionales.

- Ventajas para los propios mayores
- Afirmando que se sienten bien o muy bien, cuando realizan estas tareas.

- Aumentando su autoestima.

- Mejorando su calidad de vida.

Para concluir, entendemos a los adultos mayores "poco", hay que enseñarles de solidaridad, conciencia crítica, responsabilidad, activismo, compromiso y participación, entre otros. Para ello, sólo hay que conocer y desarrollar los mecanismos adecuados para encauzar estas potencialidades hacia la Educación para el Desarrollo, con el objetivo de lograr un mundo más justo.

\section{Referencias bibliográficas}

Amador, L. (2009). Los programas universitarios de mayores y el envejecimiento activo. En el IX Congreso Nacional de Organizaciones de Mayores (CEOMA) Comunicación nº 54.

Argibay, M. \& Celorio, G. (2005). La Educación para el Desarrollo. Vitoria-Gasteiz: Servicio Central de Publicaciones del Gobierno Vasco.

Argibay, M; Celorio, G. \& Celorio, J.J. (1977). Educación para el Desarrollo. El espacio olvidado para la Cooperación. Cuadernos de Trabajo, 19 Bilbao: HEOGA.

Blazquez, F. (2002). Los mayores, nuevos alumnos de la Universidad. Revista Interuniversitaria de Formación de Profesorado, 45, 89-105.

Boni, A. (2005). La educación para el desarrollo en la enseñanza universitaria como una estrategia de la cooperación orientada al desarrollo humano (Tesis Doctoral) Universidad de Valencia.

Calderón, K. (2010). La vida que enseña: nociones de educación permanente. San José C. R.: EUNED.

Calidad de Vida. Pedagogía Social. Revista Interuniversitaria, 20, 245-272.

Carbonell, M ${ }^{\mathrm{a}}$ C. \& Medina, M. (2006). Las personas mayores y el voluntariado Acciones $e$ investigaciones sociales, 1, 434. 
BÚSQUEDA - Julio / Diciembre de 2014 - No. 13 (32 - 40)

Castellón, A; Gómez, M.A. \& Martos, A. (2004). Análisis de la satisfacción en los mayores de la Universidad de Granada. Revista multidisciplinar de gerontología 14 (5), 252-257.

Cebeiro, M. (2010, 19 de enero). El mundo entero envejece. Periódico El País.

Celorio, J.J. (1995). La Educación para el Desarrollo. Cuadernos Bakeaz, 9. Bilbao: Bakeaz.

Coordinadora de ONGD España (2005). Educación para el desarrollo: una estrategia de cooperación imprescindible. Madrid: Coordinadora de ONGD España.

Delors, J. (1996). Informe Delors. La educación encierra un tesoro. Madrid: UNESCOSantillana.

Escarbajal de Haro, A. \& Martínez de Miguel, S. (2012). Jubilación, Educación y

Fernández, C., Macías, L. \& Orte, C. (2009) Qualitat de vida i educació de la gent gran. En C. Orte (Dir.) Anuari de l'envelliment. Illes Balears 2009 (215-239). Palma: Càtedra d'Atenció a la Dependència i Promoció de l'Autonomia Personal (Conselleria d'Afers Socials, Promoció i Immigració i UIB).

Fernández, C., Macías, L., Mas, C. \& Orte, C. (2008). Uso de las tecnologías de la información y la comunicación entre el alumnado de la Universitat Oberta per a Majors de les Illes Balears. En M.C. Palmero (coord.). Formación universitaria de personas mayores $y$ promoción de la autonomía personal. Políticas socioeducativas, metodologías e innovaciones (pp. 233-244). Burgos: Universidad de Burgos.

Hernández, R., Fernández, C. \& Baptista $P$ (1998). Metodología de la Investigación. México: Mc Graw Hill

IMSERSO (2010). Encuesta mayores 2010. Madrid: IMSERSO.
Lemieux, A. (1998). Los Programas Universitarios para mayores. Enseñanza e investigación. Madrid: IMSERSO.

Limón, Mª R. (2007). La educacióntransformadora, como recurso básico para vivir la vejez. Madrid: Arjé Servicios Comunitarios.

Moody, R. H.(1976). Philosophical presuppositions of education for Old Age, Educational Gerontology, 1. 1-16.

Pérez Díaz, J. (2003). ¿Cómo ha mejorado tanto la vejez en España? En CEDDAR, II ${ }^{\mathrm{s}}$ Jornadas sobre Políticas Demográficas y de Población (pp. 81-107). Zaragoza: Gobierno de Aragón.

Pérez Serrano, G. (2001). Aprender a lo largo de la vida. Desafío de la sociedad actual. Agora digital, 1.

Requejo, A. (1999). Educación de adultos: Programas Universitarios para personas mayores. ADAXE Revista de estudios y experiencias educativas. 14-15. 109-130

Rodríguez-Díez, J.L, Amador, L.V., Pérez De Guzmán, V. \& Esteban, M. (2012). Nunca es tarde para aprender. Los Programas Universitarios para Mayores. Decápolis, 4. 60-63.

Villaplana, C. (2010). Relación entre los programas universitarios para Mayores, la satisfacción durante la jubilación y la calidad de vida. En XIX Jornadas de la Asociación de Economía de la Educación. Zaragoza: AEDE y Universidad de Zaragoza.

Vives, M., Macias, L. \& Orte, C. (2004). El apoyo social de los alumnos de la Universitat Obertaper a Majors. EnC. Orte\& M. Gambús (eds.). Los Programas Universitarios para Mayores en la Construcción del Espacio Europeo de Enseñanza Superior (pp. 317321). Palma: Universitat de les Illes Balears.

VV.AA. (2010). El Libro Blanco del Envejecimiento Activo. Sevilla: Consejería para la Igualdad y Bienestar Social. 